\title{
StentBoost Visualization for the Evaluation of Coronary Stent Expansion During Percutaneous Coronary Interventions
}

\author{
Fernando Cura $\cdot$ Mariano Albertal $\cdot$ Alfonsina Candiello • \\ Gerardo Nau • Victor Bonvini · Hernan Tricherri • \\ Lucio T. Padilla $\cdot$ Jorge A. Belardi
}

To view enhanced content go to www.cardiologytherapy-open.com

Received: September 23, 2013 / Published online: December 6, 2013

(c) The Author(s) 2013. This article is published with open access at Springerlink.com

\section{ABSTRACT}

Introduction: Inadequate stent implantation is associated with stent thrombosis and restenosis. StentBoost can enhance stent visualization and evaluate stent expansion. Currently, there are limited comparison studies between StentBoost and intravascular ultrasound (IVUS). We aimed to test the correlation and agreement between IVUS and StentBoost measurements.

Methods: From December 2010 to December 2011, 38 patients (54 stents) were analyzed

Electronic supplementary material The online version of this article (doi:10.1007/s40119-013-0023-2) contains supplementary material, which is available to authorized users.

F. Cura $(\bowtie) \cdot$ M. Albertal · A. Candiello · G. Nau . V. Bonvini · H. Tricherri - L. T. Padilla · J. A. Belardi Department of Interventional Cardiology and Endovascular Therapeutics, Instituto Cardiovascular de Buenos Aires, Buenos Aires, Argentina e-mail: fcura@icba.com.ar

M. Albertal

e-mail: marianoalbertal@gmail.com using IVUS and StentBoost. Minimal stent diameter and proximal and distal edge stent diameter were compared between imaging techniques using Pearson correlation and Bland-Altman scatter plot.

Results: There was good correlation between StentBoost and IVUS measurements regarding minimal stent diameter $(p<0.001$ in all stent portions) and an optimal agreement between IVUS and StentBoost, while lesser agreement was found between IVUS and quantitative coronary angiography.

Conclusion: The assessment of stent implantation using StentBoost showed an adequate correlation and agreement with IVUS. This easily applicable angiographic technique can be used to guide stent implantation.

Keywords: Angiography; Cardiology; Intravascular ultrasound (IVUS); Percutaneous coronary intervention; Quantitative angiography (QCA); StentBoost

\section{INTRODUCTION}

Percutaneous coronary intervention (PCI) with stent implantation constitutes the most 
common form of coronary revascularization, improving quality of life and even clinical outcome in certain patient subsets [1, 2]. Advances in stent technology have improved PCI results; however, acute (stent thrombosis) and late (in-stent restenosis and thrombosis) complications still occur [3-5]. Quantitative angiography (QCA) demonstrated that the presence of stent underexpansion contributes to restenosis and stent thrombosis [6]. Furthermore, the use of intracoronary ultrasound (IVUS) enables a more precise assessment of stent expansion than QCA and identifies stent malapposition. Several IVUS studies have shown that the presence of insufficient stent expansion and malapposition remain strong predictors of stent thrombosis [711]; however, this invasive imaging technique is poorly reimbursed in many regions, increases procedural complexity and cost and is associated with significant center-to-center variations $[12$, 13]. Indeed, the use of IVUS requires technical expertise to perform and interpret the results.

The StentBoost (Philips Medical Systems, Eindoven, The Netherlands) is a novel imaging technique that augments the fluoroscopic visualization of stents [14]. Through superimposing motion-corrected acquisition frames, a superb-quality image of an already deployed stent can be created.

The aim of this study is to evaluate the complementary value of using StentBoost to improve the assessment of stent dimensions in addition to standard QCA by comparing the correlation of stent diameter with the goldstandard measurements by IVUS.

\section{METHODS}

This prospective, single-center cohort study included patients with known obstructive coronary artery disease undergoing PCI. Inclusion criteria included patients 18 years old and a clinical indication for stent implantation. Exclusion criteria included refusal to participate in the study, implantation of a stent for in-stent restenosis, ST-segment elevation myocardial infarction, emergent procedure, extreme tortuosity or very small vessels precluding the use of IVUS. Patients with bifurcations treated with double stent technique, severely calcified lesion or lesions that required more than one stent were also excluded. All procedures followed were in accordance with the ethical standards of the responsible committee on human experimentation (institutional and national) and with the Helsinki Declaration of 1975, as revised in 2000 and 2008. Informed consent was obtained from all patients for being included in the study.

All patients underwent PCI completely in accordance with the normal routine from December 2010 to December 2011. Thirty-eight patients underwent PCI and were included in the present analysis. All clinical decisions regarding the procedure were made by the attending operator on the basis of his interpretation of the clinical and procedural data. By study protocol, StentBoost and IVUS were performed after stent deployment after results were judged appropriate by visual assessment of coronary angiography. Then, IVUS measurements of the reference vessel and stented segment were made real time and were available for procedural decision making. Quantitative analysis of angiography and quantitative StentBoost were only performed off-line post-procedure and did not influence clinical care.

\section{Quantitative Coronary Analysis}

Acquisition of all angiographic images was obtained with a digital flat-panel cardiac imaging 
system (Allura Xper FD 20, Philips Medical Systems, Bothell, WA, USA). QCA analysis was performed using a previously validated, commercially available semi-automated QCA package (Inturis; Philips Medical Systems, Eindoven, The Netherlands) that incorporates both edgedetection and densitometric algorithms. Frames for QCA analysis were selected from fully opacified angiograms that provided optimal visualization of the lesion-treated segment with the least degree of foreshortening. Calibration was performed with the use of the contrast-filled guiding catheter as the reference. Post-PCI, minimal stent diameter and stent diameter at the proximal and distal edges of the stent were obtained.

\section{Intravascular Ultrasound}

After infusion of $200 \mu \mathrm{g}$ of intracoronary nitroglycerin, IVUS was performed using a 2.9 French $40 \mathrm{MHz}$ rotational catheter (Atlantis ${ }^{\mathrm{TM}}$ Pro; Boston Scientific, Natick, MA, USA) with an automated pullback at $0.5 \mathrm{~mm} / \mathrm{s}$. IVUS was performed after stent implantation or postdilation when necessary per study protocol. Quantitative IVUS analysis was performed using a commercially available software package (Galaxy; Boston Scientific, Natick, MA,
USA). Minimal stent area, minimal stent diameter as well as stent diameter for the proximal and distal edges were recorded. The percent area expansion was defined as the minimum stent area divided by the average of the proximal and distal reference areas. In addition, the presence of edge dissections or hematoma at the stent edges was also recorded.

\section{StentBoost Analysis}

StentBoost was performed using a flat-panel detector that was positioned to minimize stent foreshortening. With the guiding catheter in the field and the balloon markers located within the stented segment, roughly 40 frames of digital cine $(3 \mathrm{~s})$, were acquired without injection of contrast at 15 frames per second (Fig. 1). The portion of the frame that includes the stent was then selected as a region of interest. Automatic calibration allows measurement of stent diameters. Similar to QCA, we measured minimal and maximal stent diameter and two additional stent diameters (proximal and distal). The following comparisons were made: minimal and maximal stent diameter on IVUS to StentBoost and QCA; and proximal and distal stent diameter on IVUS to StentBoost and QCA.

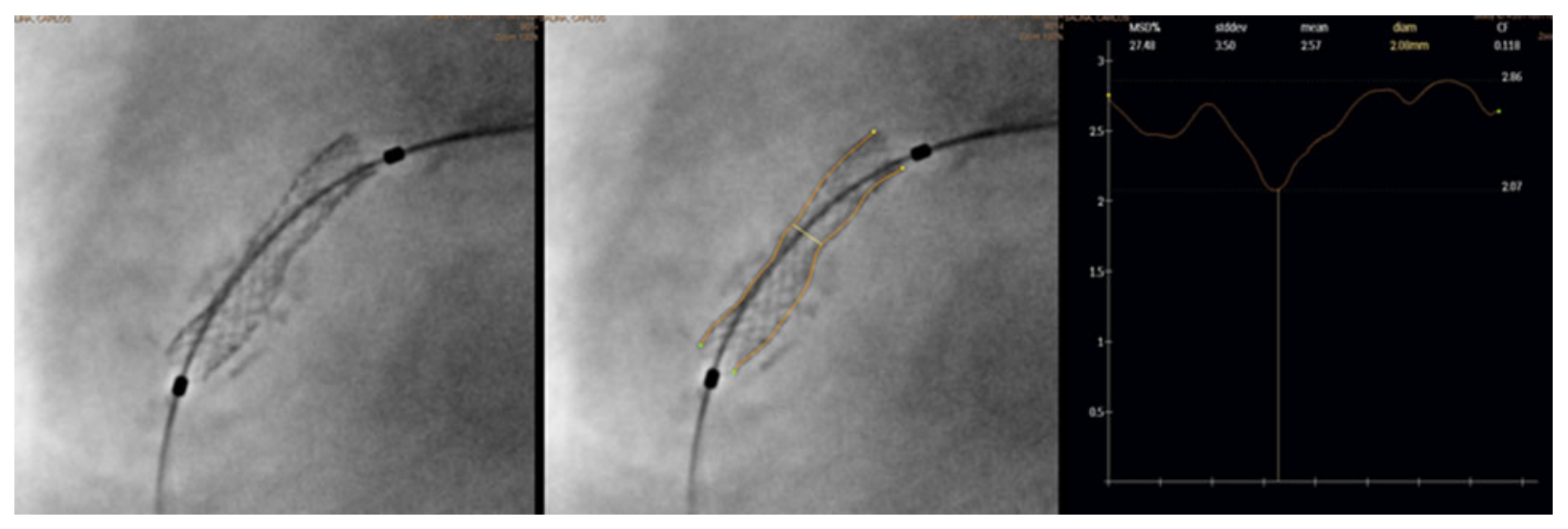

Fig. 1 StentBoost enhanced stent image (left). Same image with outlined stent borders (center) StentBoost quantitative analysis (right) shows a minimal stent diameter of $2.07 \mathrm{~mm}$ 


\section{Statistical Analysis}

Data are presented as mean values and standard deviations for continuous variables. Differences between QCA, IVUS, and StentBoost measurements were compared using the Pearson product-moment correlation and Bland-Altman analysis. All analyses were performed using SPSS (version 17.0, IBM, Armonk, New York, USA).

\section{RESULTS}

Baseline characteristics are detailed in Table 1. A total of 54 lesions were stented and comprised $3.8 \%$ of the left main, $33.3 \%$ of the left anterior descending, $29.6 \%$ of the left circumflex/acute marginal branch and $33.3 \%$ of the right coronary artery. Of the 54 stents implanted, $42.6 \%$ were Liberte (Boston Scientific, Natick, MA, USA), 16.7\% Cypher (Cordis Corporation, Johnson \& Johnson, Warren, NJ, USA) $16.7 \%$ Resolute (Medtronic, Inc., Santa Rosa, CA, USA), 12.0\% Taxus (Boston Scientific, Natick, MA, USA) and 12.0\% Presillion (Cordis Corporation, Johnson \&

Table 1 Baseline characteristics

\begin{tabular}{ll}
\hline Variable & $n=38$ \\
\hline Age (years) & $64.5 \pm 8.7$ \\
Females & $5(13.2)$ \\
Hypertension & $30(78.9)$ \\
Diabetes & $6(15.8)$ \\
Current smokers & $6(15.8)$ \\
Dyslipidemia & $32(84.2)$ \\
Prior myocardial infarction & $7(18.4)$ \\
Prior coronary bypass grafting & $7(18.4)$ \\
Prior percutaneous coronary intervention & $24(63.2)$ \\
\hline
\end{tabular}

Continuous variables are presented as mean of six standard deviations. Dichotomous variables are presented as numbers (\%)
Johnson, Warren, NJ, USA). The average stent diameter was $3.2 \pm 0.5 \mathrm{~mm}$, the stent length was $15.8 \pm 12.0 \mathrm{~mm}$, and the stents were deployed at a mean pressure of $15.2 \pm 3.0 \mathrm{~atm}$. From 54 lesions, $57.4 \%$ were type A, $20.4 \%$ were type B1/ 2 and $22.2 \%$ were type C. Pre-dilation and postdilatation were performed in 50.0 and $33.3 \%$ of the lesions (Fig. 2), respectively.

\section{QCA and StentBoost Measurements}

Quantitative angiography measurements were performed in all 38 patients after placement of 54 stents. The mean percent diameter stenosis by QCA before intervention was $73 \pm 11 \%$. The mean lesion length was $14 \pm 5 \mathrm{~mm}$ and vessel size was $3.25 \pm 0.50 \mathrm{~mm}$. After stent delivery and/or post-dilation, the minimal stent diameter by QCA was $2.76 \pm 0.53 \mathrm{~mm}$ (Table 2) and by StentBoost $2.74 \pm 0.49 \mathrm{~mm}$. Stent expansion was $90 \pm 8 \%$ by QCA and $88 \pm 8 \%$ by StentBoost.

\section{IVUS Measurements}

Intravascular ultrasound measurements were performed in all 54 stents. The minimal stent diameter was $3.10 \pm 0.51 \mathrm{~mm}$ (Table 2). The percentage of stent expansion was $84 \pm 8 \%$. Proximal and distal reference segment luminal diameter was $3.60 \pm 0.70$ and $3.30 \pm 0.50 \mathrm{~mm}$, respectively. Minimal stent area was $7.70 \pm 2.90 \mathrm{~mm}^{2}$.

\section{Comparison of Stent Expansion by QCA, StentBoost and IVUS}

Correlation coefficients, $r$-value and associated $p$ values for all comparisons are given in Table 3 . For minimal stent diameter assessment, IVUS, StentBoost and QCA had optimal correlation, whereas correlations were slightly weaker for stent diameters at both edges (Table 3). 

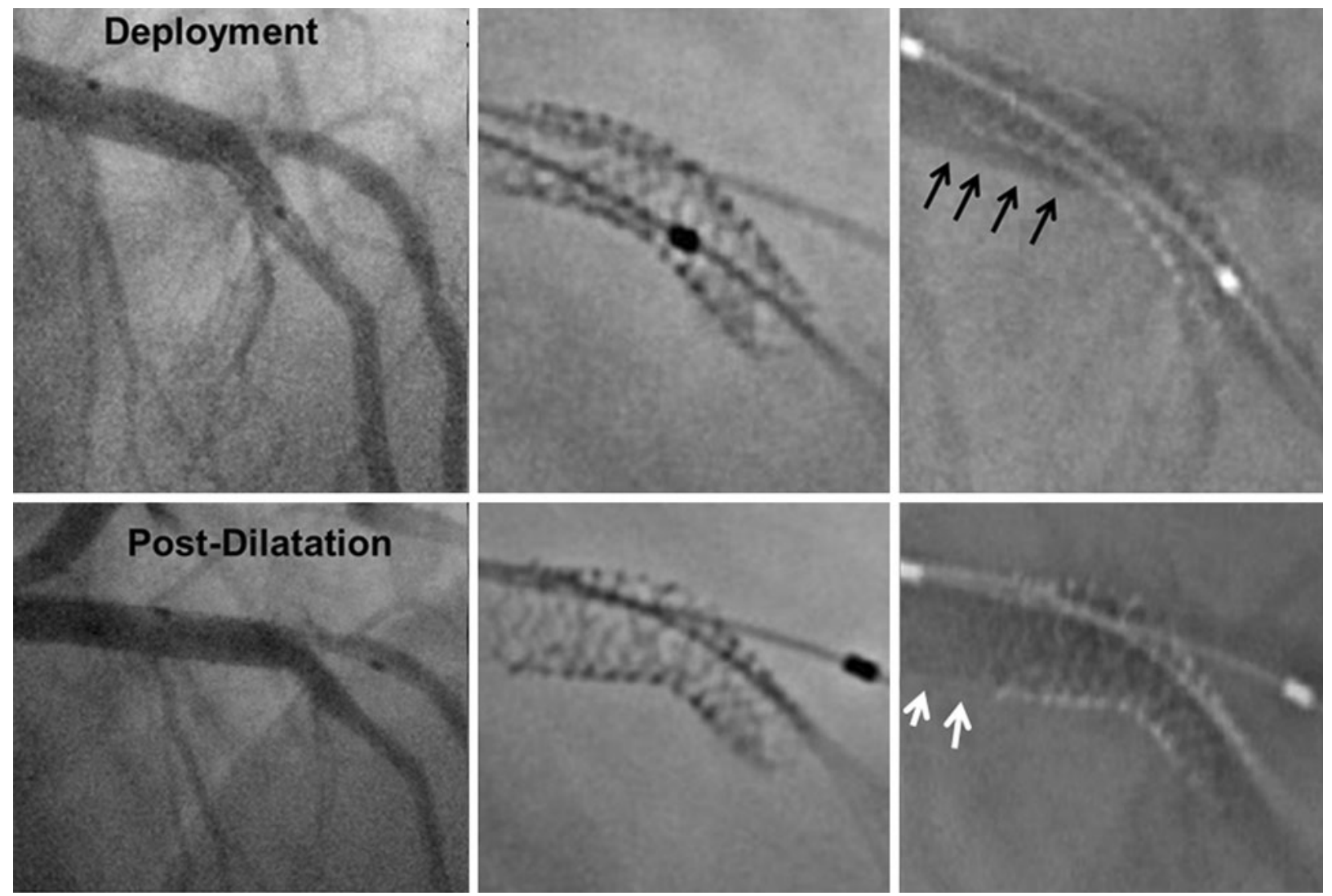

Fig. 2 StentBoost enhanced stent images. Above Note diffuse stent underexpansion (black arrows) after deployment that was corrected after balloon post-dilatation. Below

Table 2 In-stent measurements by intravascular ultrasound (IVUS), quantitative angiography (QCA) and StentBoost

\begin{tabular}{llll}
\hline & QCA & SB & IVUS \\
\hline MSD & $2.76 \pm 0.53$ & $2.74 \pm 0.49$ & $3.10 \pm 0.51$ \\
MXSD & $3.24 \pm 0.57$ & $3.18 \pm 0.58$ & $3.61 \pm 0.59$ \\
ASD & $3.02 \pm 0.53$ & $2.96 \pm 0.51$ & $3.33 \pm 0.52$ \\
Diameter ratio $^{a}$ & $0.15 \pm 0.07$ & $0.13 \pm 0.08$ & $0.16 \pm 0.10$ \\
\hline
\end{tabular}

$A S D$ average stent diameter, IVUS intravascular ultrasound, $M S D$ minimal stent diameter, $M X S D$ maximum stent diameter, $Q C A$ quantitative angiography, $S B$ StentBoost

${ }^{a}$ MXSD - MSD/MXSD

Correlation between StentBoost and IVUS did not vary according to vessel size. Bland-Altman analysis revealed a good agreement between
Note stent underexpansion focally in the lateral branch (white arrows) successfully corrected with balloon dilation

StentBoost and IVUS for minimal stent diameter assessment and, in comparison, a lesser agreement between QCA and IVUS (Figs. 3, 4). Percent stent expansion by IVUS did not correlate with the one derived by either QCA or StentBoost.

\section{DISCUSSION}

Procedural parameters such as total stent length and the degree of stent expansion have been associated with increased risk of restenosis and thrombosis following PCI $[7,8]$. A number of studies have proposed different IVUS cutoffs (i.e., minimal stent cross-sectional area or diameter), which in turn have predicted repeat revascularizations after drug-eluting stent DES and bare-metal stent deployment $[4,15,16]$. 
Table 3 Comparison of measurements by intravascular ultrasound (IVUS), quantitative angiography(QCA) and StentBoost

\begin{tabular}{|c|c|c|}
\hline$(N=54)$ & $R(p$ value $)$ & $\begin{array}{l}\text { Absolute } \\
\text { difference } \\
(\mathrm{mm})\end{array}$ \\
\hline $\begin{array}{l}\text { MXSD by IVUS and } \\
\text { MXSD by SB }\end{array}$ & $0.70(<0.001)$ & $0.43 \pm 0.45$ \\
\hline $\begin{array}{l}\text { MXSD by IVUS and } \\
\text { MXSD by QCA }\end{array}$ & $0.73(<0.001)$ & $0.34 \pm 0.35$ \\
\hline $\begin{array}{l}\text { MSD by IVUS and } \\
\text { MSD by SB }\end{array}$ & $0.68(<0.001)$ & $0.38 \pm 0.06$ \\
\hline $\begin{array}{l}\text { MSD by IVUS and } \\
\text { MSD by QCA }\end{array}$ & $0.64(<0.001)$ & $0.44 \pm 0.07$ \\
\hline $\begin{array}{l}\text { ASD by IVUS and } \\
\text { ASD by SB }\end{array}$ & $0.68(<0.001)$ & $0.36 \pm 0.42$ \\
\hline $\begin{array}{l}\text { ASD by IVUS and } \\
\text { ASD by QCA }\end{array}$ & $0.72(<0.001)$ & $0.28 \pm 0.36$ \\
\hline $\begin{array}{l}\text { PSD by IVUS and PSD } \\
\text { by SB }\end{array}$ & $0.65(<0.001)$ & $0.39 \pm 0.51$ \\
\hline $\begin{array}{l}\text { PSD by IVUS and PSD } \\
\text { by QCA }\end{array}$ & $0.70(<0.001)$ & $0.29 \pm 0.50$ \\
\hline $\begin{array}{l}\text { DSD by IVUS and } \\
\text { DSD by SB }\end{array}$ & $0.65(<0.001)$ & $0.39 \pm 0.50$ \\
\hline $\begin{array}{l}\text { DSD by IVUS and } \\
\text { DSD by QCA }\end{array}$ & $0.65(<0.001)$ & $0.36 \pm 0.49$ \\
\hline
\end{tabular}

$A S D$ average stent diameter, DSD stent diameter, IVUS intravascular ultrasound, $M S D$ minimal stent diameter, $M X S D$ maximum stent diameter, PSD proximal stent diameter, $Q C A$ quantitative angiography, $S B$ StentBoost

Furthermore, the use of IVUS enables recognition of causes of early stent thrombosis such as the presence of stent fracture, underexpansion or inflow/outflow problems [17-19]. Notably, despite the advantages of the use of IVUS, its routine use varies considerably between centers. The use of IVUS adds additional cost and time to procedures, and requires training of operators and staff. In addition, although rare, complications related to IVUS have been reported [20]. StentBoost is an adjunctive imaging technique that amplifies fluoroscopic visualization of stents specifically and is designed to guide stent deployment. With StentBoost, the stent can be evaluated in a qualitative fashion (i.e., evaluation of stent integrity and symmetry) and in a quantitative objective fashion (i.e., stent diameter at different segments and in different projections [21]. In contrast, QCA assessment quantifies luminal dimension following contrast coronary injection; however, it does not directly determine stent dimensions. Thus, QCA and StentBoost provide additive information and constitute complementary imaging tools during PCI. In the present study, the assessment of minimal stent diameter by StentBoost had a good correlation and agreement with IVUS. The results of this study are in line with a previous one that included 30 patients who underwent IVUS, StentBoost and QCA [14]. In the aforementioned study, minimal stent diameter by IVUS strongly correlated with StentBoost ( $r=0.75, P<0.0001)$, while a good correlation was found with QCA $(r=0.65, P<0.0001)$. Despite the optimal correlation and agreement found in our study, the absolute differences in minimal stent diameter values were not negligible $(0.38 \pm 0.06 \mathrm{~mm})$. IVUS allows circumferential luminal assessment, while StentBoost is only two dimensional. Possibly, the use of multiple orthogonal views may increase StentBoost accuracy, but it may increase X-ray exposure [22]. Contrary to IVUS, the use of StentBoost does not significantly prolong the procedure, since the insertion of an additional catheter is not needed and imaging acquisition is rather fast. Although StentBoost requires additional X-ray exposure, this increment is minimal with respect to standard PCI and is not likely to be clinically relevant.

In the present study, the limitations include the lack of stent area data collection by QCA or StentBoost and only diameters were compared. 

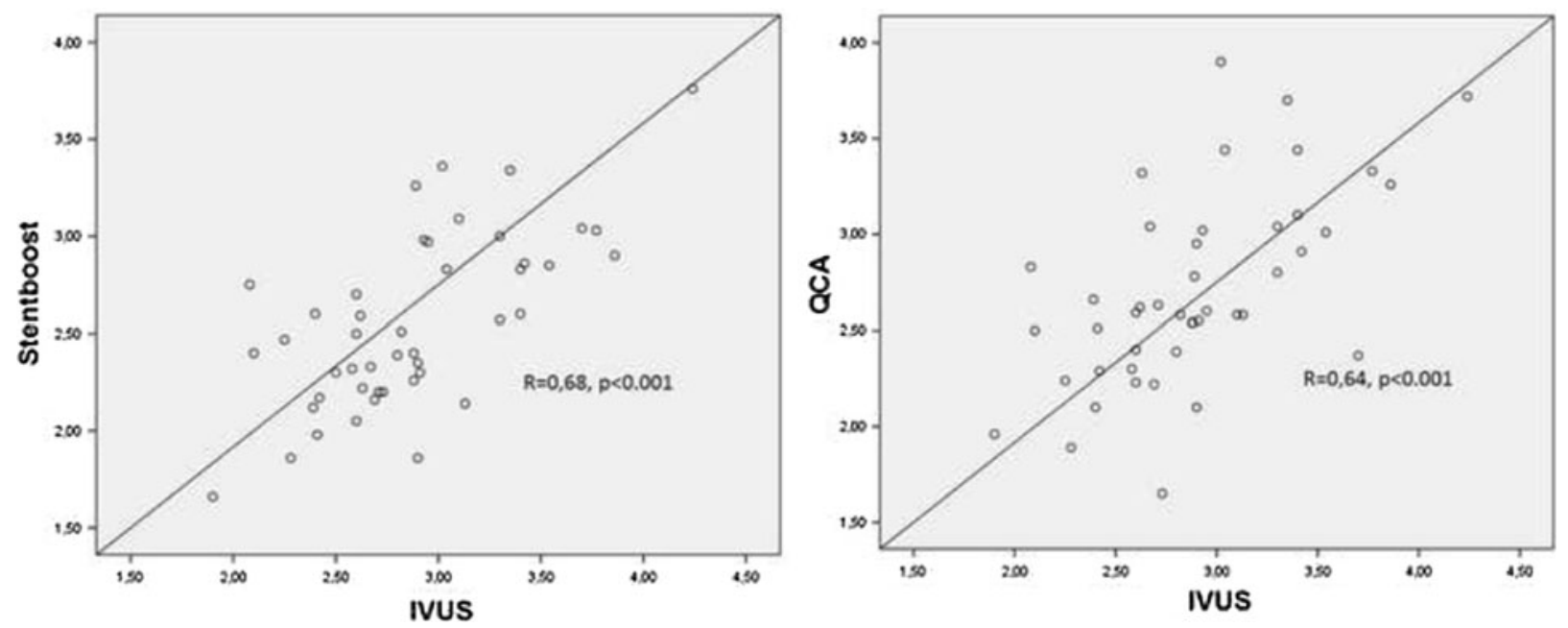

Fig. 3 Scatter plots comparing minimal stent diameter: left StentBoost and intravascular ultrasound (IVUS) measurements, right quantitative angiography (QCA)and IVUS
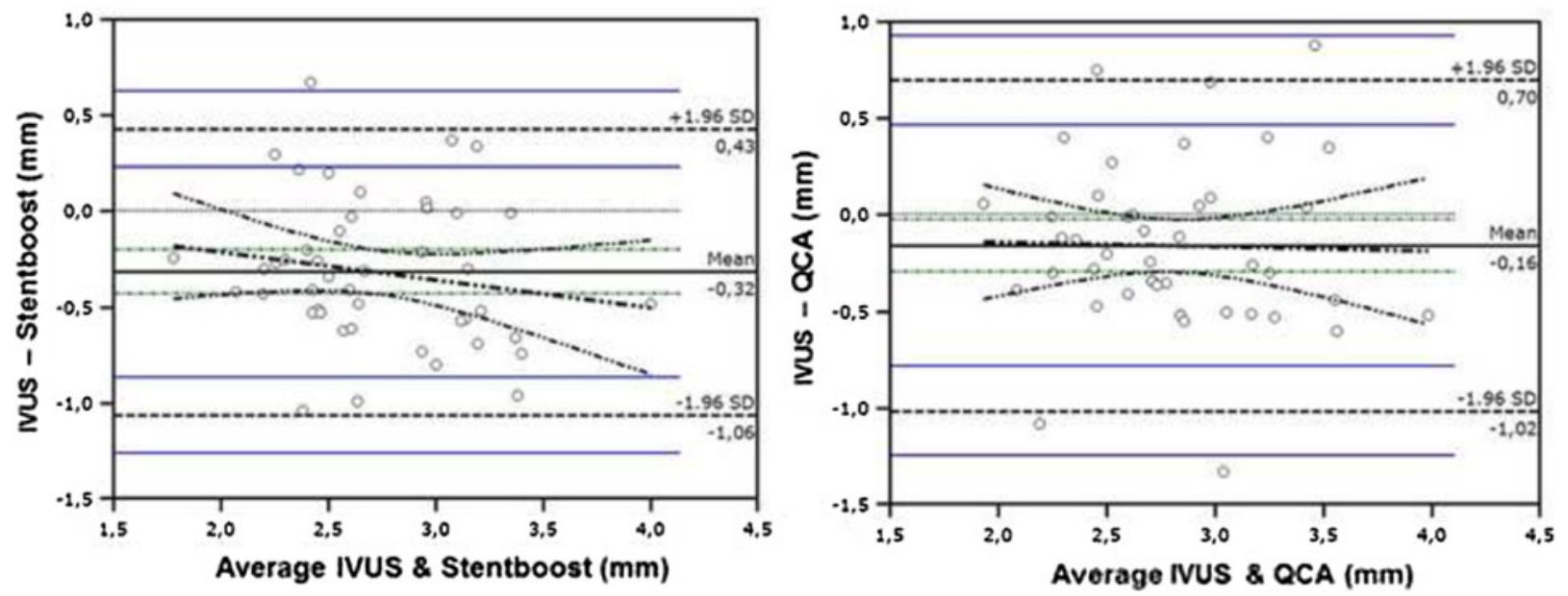

Fig. 4 Bland-Altman analysis demonstrates optimal agreement in minimal stent diameter assessment between StentBoost and IVUS and suboptimal agreement between QCA and IVUS

Recent innovation in StentBoost technique allows concomitant coronary injection while acquiring StentBoost images. This combination of luminogram and StentBoost or so called StentBoost subtraction is capable of identifying discrepancies in QCA and StentBoost dimensions, because of either stent malapposition or plaque prolapse. Figure 5 demonstrates the value of contrasted
StentBoost showing an underdeployed stent, which was unnoticed by traditional angiography.

In conclusion, this single-center cohort study evaluated the role of StentBoost during stent implantation assessment. Minimal stent diameter measured using StentBoost, IVUS and QCA demonstrated good correlation and agreement, with the highest agreement 


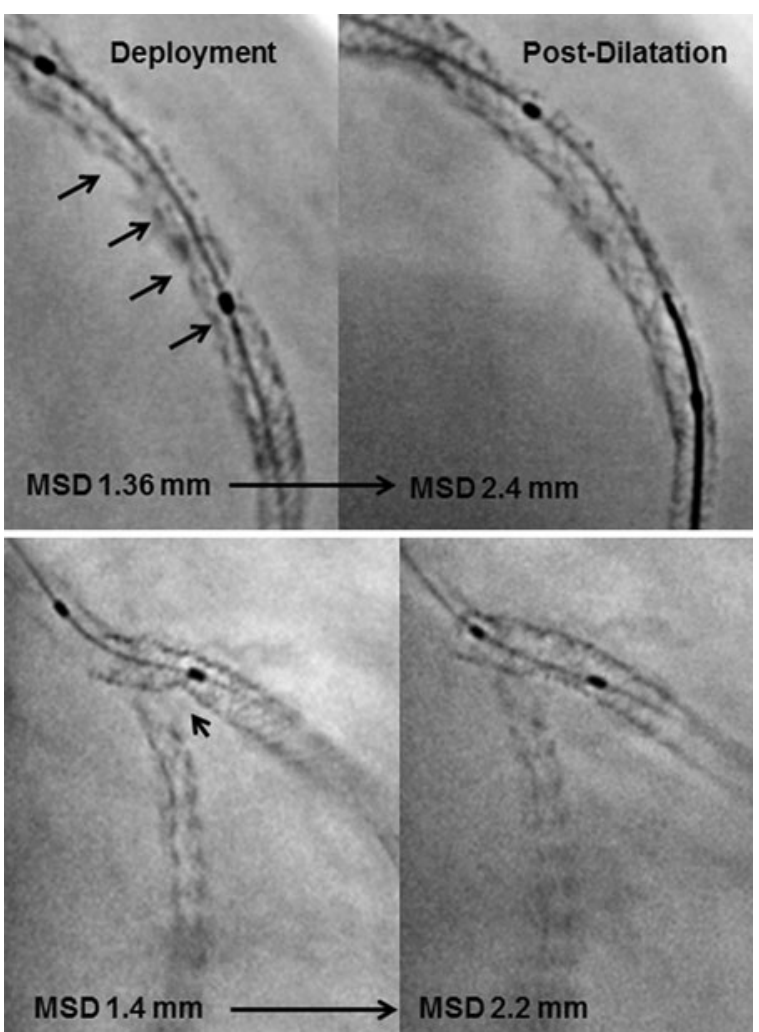

Fig. 5 Above Bifurcated left anterior descending coronary artery lesion treated with a single stent at the main vessel. Coronary angiography shows an optimal result after stent deployment and by standard StentBoost, while contrasted StentBoost image demonstrates contrast opacification outside the stent, compatible with stent malapposition. Below Optimal angiographic, standard and contrasted StentBoost results are observed after adequate balloon post-dilation

between StentBoost and IVUS. Further research is warranted to define the role of StentBoost in patients undergoing more complex PCI. Future advances in three-dimensional image could improve StentBoost diagnostic accuracy, especially in cases with complex coronary anatomy.

\section{ACKNOWLEDGMENTS}

Dr. Albertal is the guarantor for this article and takes responsibility for the integrity of the work as a whole. No funding or sponsorship was received for this study or publication of this article.

Conflict of interest. Fernando Cura, Mariano Albertal, Alfonsina Candiello, Gerardo Nau, Victor Bonvini, Hernan Tricherri, Lucio T. Padilla and Jorge A. Belardi declare no conflict of interest.

Compliance with ethics guidelines. All procedures followed were in accordance with the ethical standards of the responsible committee on human experimentation (institutional and national) and with the Helsinki Declaration of 1975 , as revised in 2000 and 2008. Informed consent was obtained from all patients for being included in the study.

Open Access. This article is distributed under the terms of the Creative Commons Attribution Noncommercial License which permits any noncommercial use, distribution, and reproduction in any medium, provided the original author(s) and the source are credited.

\section{REFERENCES}

1. Anderson JL, Adams CD, Antman EM, Bridges CR, Califf RM, Casey DE Jr, et al. 2012 ACCF/AHA focused update incorporated into the ACCF/AHA 2007 guidelines for the management of patients with unstable angina/non-ST-elevation myocardial infarction: a report of the American College of Cardiology Foundation/American Heart Association Task Force on Practice Guidelines. J Am Coll Cardiol. 2013;61:e179-347.

2. Levine GN, Bates ER, Blankenship JC, Bailey SR, Bittl JA, Cercek B, et al. 2011 ACCF/AHA/SCAI guideline for percutaneous coronary intervention: a report of the American College of Cardiology Foundation/American Heart Association Task Force on Practice Guidelines and the Society for Cardiovascular Angiography and Interventions. Catheter Cardiovasc Interv. 2013;82:E266-355.

3. Kawaguchi R, Angiolillo DJ, Futamatsu H, Suzuki N, Bass TA, Costa MA. Stent thrombosis in the era of 
drug eluting stents. Minerva Cardioangiol. 2007;55:199-211.

4. Choi SY, Maehara A, Cristea E, Witzenbichler B, Guagliumi G, Brodie B, et al. Usefulness of minimum stent cross sectional area as a predictor of angiographic restenosis after primary percutaneous coronary intervention in acute myocardial infarction (from the HORIZONS-AMI Trial IVUS substudy). Am J Cardiol. 2012;109:455-60.

5. Kang SJ, Ahn JM, Song H, Kim WJ, Lee JY, Park DW, et al. Comprehensive intravascular ultrasound assessment of stent area and its impact on restenosis and adverse cardiac events in 403 patients with unprotected left main disease. Circ Cardiovasc Interv. 2011;4:562-9.

6. Serruys PW, Kay IP, Disco C, Deshpande NV, de Feyter PJ. Periprocedural quantitative coronary angiography after Palmaz-Schatz stent implantation predicts the restenosis rate at six months: results of a meta-analysis of the BElgian NEtherlands Stent study (BENESTENT) I, BENESTENT II Pilot, BENESTENT II and MUSIC trials. Multicenter Ultrasound Stent In Coronaries. J Am Coll Cardiol. 1999;34:1067-74.

7. Cheneau E, Leborgne L, Mintz GS, Kotani J, Pichard $A D$, Satler LF, et al. Predictors of subacute stent thrombosis: results of a systematic intravascular ultrasound study. Circulation. 2003;108:43-7.

8. Choi SY, Witzenbichler B, Maehara A, Lansky AJ, Guagliumi G, Brodie B, et al. Intravascular ultrasound findings of early stent thrombosis after primary percutaneous intervention in acute myocardial infarction: a Harmonizing Outcomes with Revascularization and Stents in Acute Myocardial Infarction (HORIZONS-AMI) substudy. Circ Cardiovasc Interv. 2011;4:239-47.

9. Fujii K, Carlier SG, Mintz GS, Yang YM, Moussa I, Weisz G, et al. Stent underexpansion and residual reference segment stenosis are related to stent thrombosis after sirolimus-eluting stent implantation: an intravascular ultrasound study. J Am Coll Cardiol. 2005;45:995-8.

10. Moussa I, Di Mario C, Reimers B, Akiyama T, Tobis $\mathrm{J}$, Colombo A. Subacute stent thrombosis in the era of intravascular ultrasound-guided coronary stenting without anticoagulation: frequency, predictors and clinical outcome. J Am Coll Cardiol. 1997;29:6-12.

11. Okabe T, Mintz GS, Buch AN, Roy P, Hong YJ, Smith KA, et al. Intravascular ultrasound parameters associated with stent thrombosis after drug-eluting stent deployment. Am J Cardiol. 2007;100:615-20.
12. Mueller C, Hodgson JM, Schindler C, Perruchoud AP, Roskamm H, Buettner HJ. Cost-effectiveness of intracoronary ultrasound for percutaneous coronary interventions. Am J Cardiol. 2003;91:143-7.

13. Schiele F, Meneveau N, Seronde MF, Caulfield F, Pisa B, Arveux P, et al. Medical costs of intravascular ultrasound optimization of stent deployment. Results of the multicenter randomized 'REStenosis after Intravascular ultrasound STenting' (RESIST) study. Int J Cardiovasc Interv. 2000;3:207-13.

14. Mishell JM, Vakharia KT, Ports TA, Yeghiazarians Y, Michaels AD. Determination of adequate coronary stent expansion using StentBoost, a novel fluoroscopic image processing technique. Catheter Cardiovasc Interv. 2007;69:84-93.

15. McDaniel MC, Eshtehardi P, Sawaya FJ, Douglas JS Jr, Samady H. Contemporary clinical applications of coronary intravascular ultrasound. JACC Cardiovasc Interv. 2011;4:1155-67.

16. Doi H, Maehara A, Mintz GS, Yu A, Wang H, Mandinov L, et al. Impact of post-intervention minimal stent area on 9-month follow-up patency of paclitaxel-eluting stents: an integrated intravascular ultrasound analysis from the TAXUS IV, V, and VI and TAXUS ATLAS Workhorse, Long Lesion, and Direct Stent Trials. JACC Cardiovasc Interv. 2009;2:1269-75.

17. Kosonen P, Vikman S, Jensen LO, Lassen JF, Harnek $\mathrm{J}$, Olivecrona GK, et al. Intravascular ultrasound assessed incomplete stent apposition and stent fracture in stent thrombosis after bare metal versus drug-eluting stent treatment the Nordic Intravascular Ultrasound Study (NIVUS). Int J Cardiol. 2013;168:1010-6.

18. Doi H, Maehara A, Mintz GS, Tsujita K, Kubo T, Castellanos C, et al. Classification and potential mechanisms of intravascular ultrasound patterns of stent fracture. Am J Cardiol. 2009;103:818-23.

19. Park JS, Shin DG, Kim YJ, Hong GR, Cho IH. Acute myocardial infarction as a consequence of stent fracture and plaque rupture after sirolimus-eluting stent implantation. Int J Cardiol. 2009;134:e79-81.

20. Alfonso F, Flores A, Escaned J, Sanmartin M, Hernandez R, Fernandez-Ortiz A, et al. Pressure wire kinking, entanglement, and entrapment during intravascular ultrasound studies: a potentially dangerous complication. Catheter Cardiovasc Interv. 2000;50:221-5.

21. Ramegowda RT, Chikkaswamy SB, Bharatha A, Radhakrishna J, Krishnanaik GB, Nanjappa MC, et al. Circumferential stent fracture: novel 
detection and treatment with the use of StentBoost. Tex Heart Inst J. 2012;39:431-4.

22. Davies AG, Conway D, Reid S, Cowen AR, Sivananthan M. Assessment of coronary stent deployment using computer enhanced X-ray images-validation against intravascular ultrasound and best practice recommendations. Catheter Cardiovasc Interv. 2013;81:419-27. 